\title{
Evaluation of the biocompatibile ionic liquid 1-methyl-3-methylimidazolium dimethylphosphite pretreatment of corn cob for improved saccharification
}

\author{
Qiang Li • Xinglin Jiang • Yucai He • Liangzhi Li • \\ Mo Xian • Jianming Yang
}

Received: 16 November 2009/Revised: 28 January 2010 /Accepted: 29 January 2010 /Published online: 25 February 2010

(C) Springer-Verlag 2010

\begin{abstract}
Ionic liquid (IL) pretreatment of lignocellulose materials is a promising process in biomass conversion to renewable biofuel. More in-depth research involving environment-friendly IL is much needed to explore pretreatment green route. In our case, IL 1-methyl-3-methylimidazolium dimethylphosphite ([Mmim]DMP) was chosen as an environment-friendly solvent to pretreat corn cob in view of its biocompatibility with both lignocellulose solubility and cellulase activity. The pretreatment/saccharification process and in situ saccharification process involving [Mmim]DMP were efficiently performed in bioconversion of corn cob to sugars, and more than $70 \%$ saccharification rates were obtained. Furthermore, the fermentability of reducing sugars obtained from the hydrolyzates was evaluated using Rhodococcus opacus strain ACCC41043 (R. opacus). High lipid production $41-43 \%$ of cell dry matter was obtained after $30 \mathrm{~h}$ of cultivation. $\mathrm{GC} /$ MS analysis indicated that lipids from $R$. opacus contained mainly long-chain fatty acids with four major constituent/ oleic acid, stearic acid, palmitic acid, palmitoleic acid which are good candidates for biodiesel. These elucidated that corn cob pretreated by IL [Mmim]DMP did not bring negative effects on saccharification, cell growth, and accumulation of lipid of $R$. opacus. In conclusion, the IL [Mmim]DMP shows promise as green pretreatment solvent for cellulosic materials.
\end{abstract}

Electronic supplementary material The online version of this article (doi:10.1007/s00253-010-2484-8) contains supplementary material, which is available to authorized users.

Q. Li $\cdot$ X. Jiang $\cdot$ Y. He $\cdot$ L. Li $\cdot$ M. Xian $(\bowtie) \cdot$ J. Yang

Key Laboratory of Biofuel,

Qingdao Institute of Bioenergy and Bioprocess Technology,

Chinese Academy of Sciences,

Qingdao 266071, China

e-mail: xianmo@qibebt.ac.cn
Keywords Corn cob · Biocompatibility · Ionic liquids pretreatment - 1-Methyl-3-methylimidazolium dimethylphosphite $\cdot$ Enzymatic hydrolysis $\cdot$ Microbial lipid

\section{Introduction}

Lignocellulosic biomass is a plentiful and renewable resource for fuels and chemicals. The efficient conversion of lignocellulosic materials into biofuel is expected to be the needed breakthrough in producing affordable and renewable fuel (Peterson et al. 2007). Biofuel can be produced by fermentation from, e.g., sugars, starch, and various lignocellulosic materials such as corn cob, wood, and waste (Wackett 2008). Because the production of biofuel via fermentation from sugars and starch is more straightforward, biofuel production from lignocellulose creates additional technical challenges, particularly the need for pretreatment (Rubin 2008) and enzymatic hydrolysis (Wackett 2008). Lignocellulosic materials should ultimately be converted to sugars for fermentation. Furthermore, enzymatic saccharification and pretreatment process are considered to be the promising vehicle to break down lignocellulosic materials into fermentable sugars (Mosier et al. 2005). Subsequently, after pretreatment and enzymatic hydrolysis, cellulosic materials can be efficiently converted into biofuel by microbial fermentation (Wackett 2008). Therefore, the pretreatment process is one of the key points in biomass conversion to biofuel (Yang and Wyman 2008).

To make cellulosic materials more susceptible for hydrolysis, an effective pretreatment is required to soften its tough assembled structure of cellulose crystallinity (Heinze and Liebert 2001) and increase the cellulose porosity (Chandra et al. 2007; Galbe and Zacchi 2007). Pretreatment processes that increase the surface area accessible to cellulases and 
water are expected to generate improvements in efficiency of hydrolysis and conversion of cellulose to sugars (Sun and Cheng 2002; Zhang and Lynd 2004). As a result, different pretreatment methods can be used for enhancing the digestibility of cellulosic materials (Hendriks and Zeeman 2009; Mosier et al. 2005). However, in the traditional process, the use of organic solvents in bioprocess operations possesses a number of problems. The main concerns are the toxicity of the solvents to both the process operators and the environment and the volatile and flammable nature of these solvents which make them a potential explosion hazard (Rogers and Seddon 2003; Schmid et al. 1998).

Until recently, ionic liquids (ILs), so-called green solvents, have been confirmed to be efficient for lignocellulosic materials, such as cellulose, wood, or wheat straw (Dadi et al. 2006; Liu and Chen 2006; Diego et al. 2007). The cellulosic materials regenerated from ILs were found essentially amorphous and porous and were much more prone to degradation by cellulases ( $\mathrm{Li}$ et al. 2009; Dadi et al. 2006). Furthermore, increased rate of cellulose hydrolysis via cellulase in ionic liquids could lead to increased production of fermentable sugars that can be converted into fuels. In addition, ionic liquids involved processes are less energy demanding, easier to operate, and more environmentally friendly than current dissolution processes (Zhao et al. 2009; Rogers and Seddon 2003; Hélène and Lionel 2002).

However, more in-depth research involving environmentfriendly IL is much needed to explore pretreatment green route for resolving the challenge of ionic liquid application (Zhao et al. 2008; Rogers and Seddon 2003). As a result, recent efforts have been focused on exploration of enzymefriendly and environment-friendly ionic liquids (Roosen et al. 2008), because green solvent is promising for commercial application (Kamiya et al. 2008). Although deactivation of cellulase in the presence of low concentrations of $[\mathrm{Bmim}] \mathrm{Cl}$, a typical cellulose-treated IL, was demonstrated (Turner et al. $2003)$, up to $60 \%(v / v)$ of [Emim][OAc] and DMEAA gave to enzymatic resolution of amino acids (Zhao et al. 2006; Rayne and Mazza 2007), implying that the anionic component of ILs plays a key role in enzymatic catalysis. Moreover, Kamiya reported enzymatic saccharification process in presence of moderate concentration of IL 1-ethyl-3methylimidazolium diethylphosphate (Kamiya et al. 2008). Generally speaking, environment-friendly IL can perform green process to obtain enhanced enzymatic saccharification for following biofuel production using hydrolyzates. These exciting achievements and commercially profitable possibilities encourage us to explore the possibility of utilizing lignocellulosic hydrolyzates obtained from enzymatic hydrolysis of IL-treated cellulose to produce biofuel such as biodiesel (a typical biofuel). Therefore, it is a key point to evaluate the biocompatibility of ionic liquids and select green IL for pretreatment process.
In the case, our work mainly focused on evaluating ionic liquids as solvent to pretreat and enhance the enzymatic saccharification of lignocellulosic waste (corn cob) toward biodiesel production. Nine ILs were synthesized to pretreat corn cob. A systematic study on corn cob pretreatment, pretreatment/saccharification process and enzymatic in situ saccharification were investigated. Furthermore, the fermentability of the sugars in hydrolyzates and the IL pretreatment effect on lipid accumulation were evaluated using the lipid accumulating strain $R$. opacus. Such information will be vital for tackling the hydrolysis of more complex lignocellulosic materials and biotransform hydrolyzates obtained from enzymatic hydrolysis of ILtreated cellulose into biofuel.

\section{Materials and methods}

Materials and microbes

Corn cob was obtained in the suburb of Qingdao China in 2008. One thousand grams corn cob was milled into powder, washed three times, and dried at $60^{\circ} \mathrm{C}$ for $48 \mathrm{~h}$ in an air drier (type 202-2, Shanghai, China) for the pretreatment process. Avicel PH-101 (microcrystalline cellulose, MCC) was purchased from Sigma-Aldrich. The lipid accumulating bacterial strain is derivative of Rhodococcus opacus ACCC41043 from Agricultural Culture Collection of China.

Cellulase from Trichoderma reesei was obtained from Genencor (Shanghai, China) and was immobilized with infusorial earth (Long et al. 2007). 1-Methyl-3-methylimidazolium methylsulfate ([Mmim] $\left.\left[\mathrm{MeSO}_{4}\right]\right)$, 1-butyl-3methylimidazolium hexafluorophosphate $\left([\mathrm{Bmim}]\left[\mathrm{PF}_{6}\right]\right)$, $N, N$-dimethylethanolammonium acetate (DMEAA), $N, N$-dimethylethanolammonium lactate ([DMEA][lactate]), 1-butyl-1-methylpyrrolidinium chloride ([BmPy $] \mathrm{Cl})$, and 1-butyl-3-methylimidazolium chloride $([\mathrm{Bmim}] \mathrm{Cl}), 1$ ethyl-3-methyl imidazolium diethyl phosphate ([Emim]DEP) were synthesized as the reported preparation procedures (Huddleston et al. 2001; Zhang et al. 2008; Li et al. 2009).

Preparation of 1-methyl-3-methylimidazolium dimethylphosphite ([Mmim]DMP) and 2-ethyl-3-methylimidazolium dimethylphosphite ([Emim]DMP) were performed as the following procedure: Equal molar amounts of $\mathrm{N}$-methylimidazole or 2-ethylimidazole and corresponding trimethyl phosphate or triethyl phosphate were added to a roundbottomed flask fitted with a reflux condenser at $150^{\circ} \mathrm{C}$ for 8-10 h (Nie et al. 2006). The reactions were carried out under a nitrogen atmosphere to prevent uptake of water by the ILs. The resulting slightly yellow ionic liquid (viscous liquid at room temperature) was washed three times with diethyl ether at room temperature. The target salt was 
dissolved into chloroform containing 10\% $(v / v)$ methanol and was then passed through a column filled with neutral activated alumina. After removal of solvent by evaporation under reduced pressure, the ionic liquid thus obtained was dried in vacuum at $70^{\circ} \mathrm{C}$ for $8 \mathrm{~h}$. Ionic liquids were further analyzed by high performance liquid chromatography (HPLC) and nuclear magnetic resonance (NMR). The ${ }^{1} \mathrm{H}$ and ${ }^{13} \mathrm{C}$ NMR spectra were obtained in $\mathrm{CDCl}_{3}$ with a Bruker Ultra Shield 600 Plus instrument. The water content of IL was determined using a volumetric Aquastar Karl Fischer titrator (EM Science, Gibbstown). The purities of ILs were above $98 \%$. The water mass fraction was less than $3 \times 10^{-4}$. All other chemicals were from commercial source and of reagent grade.

\section{Selection of ILs for pretreatment of corn cob}

The solubility of lignocellulosic materials in IL was performed at $130^{\circ} \mathrm{C}$ by mixing variable weight of corn cob or MCC with $10 \mathrm{~g}$ IL. Pretreatment test of corn cob was carried out by mixing $0.3 \mathrm{~g}$ corn cob and $10 \mathrm{~g}$ IL in a 50-ml round-bottomed flask under a nitrogen atmosphere with magnetic stirring. After incubating the solution in the IL for $20 \mathrm{~min}$ at $130^{\circ} \mathrm{C}$, the pretreated corn cob powder was precipitated with deionic water. The corn cob sample was precipitated in seconds. After centrifugation, the surpernatant containing IL was removed for the IL recovery (Li et al. 2009), while the precipitate was washed with additions of deionic water. Buffer solution used in the corn cob enzymatic hydrolysis was used for the final washes, and the regenerated corn cob was dried at $60^{\circ} \mathrm{C}$ for $10 \mathrm{~h}$ in an air drier for the use of enzymatic hydrolysis.

The regenerated corn cob from ILs was hydrolyzed in a batch system. The total batch volume was $3 \mathrm{ml}$ with cellulase concentration of $30 \mathrm{FPU} \mathrm{g}^{-1}$ regenerated corn cob and dry powder of regenerated corn cob with concentration of $10 \mathrm{~g} / \mathrm{l}$. The enzymatic mixture was buffered with $50 \mathrm{mM}$ acetate acid-sodium acetate, $\mathrm{pH}$ 4.8. Enzymatic hydrolysis was carried out at $50^{\circ} \mathrm{C}$ for variable times in a reciprocating shaker bath. The enzymatic course was monitored by withdrawing samples from the supernatant periodically and measuring release of soluble reducing sugars by the DNS assay using D-glucose as a standard (Miller 1959).

\section{Selection of green cellulase-friendly ILs}

To select the IL which can conduct cellulase compatability, cellulase activity and enzymatic saccharification of untreated corn cob in the presence of 20\% $(v / v)$ nine ILs ([Mmim] DMP, [Emim]DEP, [Emim]DMP, [Mmim] $\left[\mathrm{MeSO}_{4}\right]$, DMEAA, [DMEA][lactate], [Bmim]Cl, [BmPy]Cl, or $[\mathrm{Bmim}]\left[\mathrm{PF}_{6}\right]$ ) were explored. A suspension solution of $30 \mathrm{mg}$ untreated corn cob in $3 \mathrm{ml}$ acetate acid-sodium acetate buffer solution ( $50 \mathrm{mM}, \mathrm{pH} 4.8)$ was incubated in a reciprocating shaker bath at $50^{\circ} \mathrm{C}$. The reaction was initiated by adding cellulase of 30 FPU $\mathrm{g}^{-1}$ substrates. The reaction was monitored by withdrawing samples from the supernatant periodically and measuring release of soluble reducing sugars by the DNS assay. All assays were performed in triplicate. Error bars show the standard deviation of triplicate measurements.

\section{Evaluation of [Mmim]DMP pretreatment} on saccharification

Recently, two kinds of processes were developed in ionic liquid-involved cellulosic material pretreatment and saccharification process: pretreatment/saccharification process (Dadi et al. 2006; Zhao et al. 2009) and in situ saccharification process (Kamiya et al. 2008). In our case, saccharification of corn cob was carried out by the two kinds of processes to evaluate [Mmim]DMP pretreatment on saccharification. In pretreatment/saccharification of corn cob, corn cob was pretreated by IL [Mmim]DMP for $20 \mathrm{~min}$ at $130^{\circ} \mathrm{C}$; then $10 \mathrm{~g} / \mathrm{l}$ regenerated corn cob was used as substrate for enzymatic hydrolysis. The total batch volume was $3 \mathrm{ml}$ with cellulase concentration of $30 \mathrm{FPU}^{-1}$ substrates, and enzymatic mixture was buffered with $50 \mathrm{mM}$ acetate acidsodium acetate solution $(\mathrm{pH} 4.8)$ at $50^{\circ} \mathrm{C}$ in a reciprocating shaker. On the other hand, in enzymatic in situ saccharification of corn cob, corn cob was pretreated by IL [Mmim] DMP for $20 \mathrm{~min}$ at $130^{\circ} \mathrm{C}$; then, appropriate volume acetate acid-sodium acetate buffer (50 mM, pH 4.8) was slowly added to the corn cob/IL mixture. Subsequently, the corn cob sample was immediately precipitated in the form of granule. In the saccharification of corn cob in aqueous-IL media, the volume ratio of the IL to water $(50 \mathrm{mM}$ acetate acid-sodium acetate buffer, $\mathrm{pH} 4.8)$ was varied from $10-60 \%(v / v)$, and the concentration of corn cob was kept constant at $10 \mathrm{mg} / \mathrm{ml}$ for a total reaction volume of $3 \mathrm{ml}$. The enzymatic saccharification was initiated by the addition of $30 \mathrm{FPU}$ cellulase to either the aqueous-IL mixture of corn cob in a reciprocating shaker at $50^{\circ} \mathrm{C}$ and $170 \mathrm{rpm}$.

The reaction was monitored by withdrawing samples from the supernatant periodically and measuring release of soluble reducing sugars by the DNS assay. All assays were performed in triplicate. Error bars show the standard deviation of triplicate measurements.

Production of microbial lipids using hydrolysates by $R$. opacus ACCC41043

In order to investigate fermentability of hydrolysates obtained from enzymatic hydrolysis, lipid fermentation of hydrolyzates was performed by $R$. opacus ACCC41043. After the IL pretreatment/saccharification process, the 
hydrolyzates from enzymatic hydrolysis were filtered, and immobilized cellulase was recycled for the next batch of enzymatic hydrolysis. On the other hand, after the enzymatic in situ process, the mixture of IL and hydrolyzates from enzymatic in situ hydrolysis were filtered and was then passed through a column filled with neutral $\mathrm{Al}_{2} \mathrm{O}_{3}$ and concentrated according to our recent literature (Xian et al. 2009). The hydrolyzates (containing $4.25 \mathrm{~g} / 1$ reducing sugars) were separated from the ionic liquid. Then, the hydrolyzates (recovered sugars) were used in lipid fermentation immediately without further purification.

The inoculum was prepared by transferring a loop of cells of R. opacus ACCC41043 into $50 \mathrm{ml}$ of mineral salts medium (MSM) as reported by Schlegel et al. (1961). Cells were harvested by centrifugation $(5,000 \mathrm{rpm}, 5 \mathrm{~min})$, suspended in sterilized water, and used for the following lipid fermentation. $R$. opacus ACCC41043 was grown aerobically at $30^{\circ} \mathrm{C}$ in $100 \mathrm{ml}$ MSM with hydrolyzates using hydrolyzates (containing 3.55 or $4.25 \mathrm{~g} / \mathrm{l}$ reducing sugars) as carbon source. To investigate the effect of ionic liquid residue on lipid accumulation and cell growth, 10 $15 \mathrm{~g} / 1$ (final concentration) [Mmim]DMP was added to the medium. Control experiment was performed using glucose as carbon source in MSM.

\section{Microbial oil preparation}

Cell cultures were harvested and prepared for fatty acid methyl esters (FAMEs) synthesis according to the described method (Wältermann et al. 2000). FAMEs were synthesized using IL $N$-methyl-2-pyrrolidonium methyl sulfate ([NMP] $\left.\left[\mathrm{CH}_{3} \mathrm{SO}_{3}\right]\right)$ as catalyst according to our earlier reported method (Zhang et al. 2009). Authentic standard (eicosanic acid) was spiked in as an internal standard in all samples.

\section{Analytical methods}

Fractions of the regenerated corn cob were measured according to the method of Goering and Van Soest (1970). It was composed of cellulose $44.8 \%$, hemicellulose $34.6 \%$, lignin $14.8 \%$, and others $5.8 \%$. One filter paper unit (FPU) is defined as the enzyme that releases $1 \mu \mathrm{mol}$ of glucose equivalents per minute from Whatman No. 1 filter paper. The released reducing sugars were measured by the DNS method using D-glucose as a standard. The rate of formation of soluble reducing sugars is taken as a measure of the rate of cellulose hydrolysis.

Untreated and [Mmim]DMP-treated corn cobs were analyzed by Fourier transformed IR (FTIR) through the following process: Corn cob was measured by BIO-RAD FTS-3500 spectrometer. The spectra $\left(4000-400 \mathrm{~cm}^{-1}\right)$ were recorded with a resolution of $4 \mathrm{~cm}^{-1}$ and 64 scans per sample. About 2.0-mg samples were prepared by mixing with $120.0 \mathrm{mg}$ of spectroscopic grade $\mathrm{KBr}$, then pressed in a standard device using a pressure of 6,000 psi to produce 13-mm diameter pellets. The background spectrum of pure $\mathrm{KBr}$ was subtracted from that of the sample spectrum.

Scanning electron microscopy (SEM) was carried out to obtain images of untreated and recovered corn cob samples from ionic liquids. The samples were coated with a thin gold layer using a vacuum sputter-coater to improve the conductivity of the samples and thus the quality of the SEM images prior to the analysis. SEM-operated at $5 \mathrm{keV}$ was used to image corn cob and cellulose samples.

Yield of reducing sugars from regenerated corn cob was calculated as follows:

Yield of released sugars $\%=$ [reducing sugars released $] \times$ [cellulose ratio in regenerated corn cob] $\times 100 /$ [regenerated corn cob weight].

The "regenerated corn cob weight" refers to the amount of the dry powder of corn cob regenerated from IL pretreatment process. Reducing sugars weight was calculated based on DNS method. As described in the fractions of the regenerated corn cob, cellulose ratio in regenerated corn cob was $44.8 \%$.

The conversion of regenerated corn cob to lipid is described as follows:

In the pretreatment/saccharification process: conversion of regenerated corn cob to lipid $\%=[$ lipid weight $] \times 100 /$ [regenerated corn cob weight].

In the enzymatic in situ hydrolysis process: conversion of regenerated corn cob to lipid \%=[lipid weight $] \times 100 \times$ $0.78 /$ [regenerated corn cob weight].

The ratio of concentration in the sugar recovery process was 0.78 (as described in the section of "Production of microbial lipids using hydrolysates by $R$. opacus ACCC41043"). "Lipid weight" refers to the weight of lipid accumulated by R. opacus ACCC41043.

Glucose released in enzymatic hydrolysis and sugar content in the hydrolysates was determined by an HPLC system equipped with a reflective index detector (Agilent1200). Inertsil $5 \mu \mathrm{m} \times 4.6 \times 250 \mathrm{~mm} \mathrm{NH}_{2}$ column was employed, and the mobile phase was consisted of acetonitrile and distilled water $(75 / 25, v / v)$ with a flow rate of $1.0 \mathrm{ml} / \mathrm{min}$. The tube temperature was $80^{\circ} \mathrm{C}$.

Fatty acid analysis

Gas Chromatography/Mass Spectrometry (GC/MS) analysis of FAMEs was done on a single quadrupole MS with an electron impact ionization source. The GC column was a 30-m long HP-5 MS (5\% phenyl)-methylpolysiloxane with an ID of $0.25 \mathrm{~mm}$ and a film thickness of $25 \mu \mathrm{m}$. The GC elution conditions were as follows: $100^{\circ} \mathrm{C}$ as the starting temperature $(5 \mathrm{~min})$, a 15 -min ramp to $250^{\circ} \mathrm{C}$, hold at $250^{\circ} \mathrm{C}$ for $10 \mathrm{~min}$. Authentic standard (eicosanic acid) was spiked in as an internal standard in all samples. 


\section{Result}

Selection of corn cob dissolving ILs with biocompatibility for pretreatment

Our work started with the screening of ILs for enhanced enzymatic hydrolysis of untreated corn cob and corn cob pretreated with ILs. Corn cob pretreated by DMEAA, [DMEA][lactate], [Bmim] $\left[\mathrm{PF}_{6}\right]$, or $[\mathrm{Mmim}]\left[\mathrm{MeSO}_{4}\right]$ which cannot conduct corn cob and MCC dissolution, appeared to result in a slightly higher saccharification rate than the untreated corn cob. After enzymatic hydrolysis of the four ILs-pretreated corn cob for $24 \mathrm{~h}$, the reducing sugar concentrations were $1.55,1.46,1.45,1.56 \mathrm{~g} / 1$ vs. $1.38 \mathrm{~g} / 1$ in buffer (Fig. 1). At the same time, corn cob dissolving ILs showed good performance in pretreatment process. ILs [Mmim]DMP, [Emim]DEP, [Emim]DMP, [Bmim]Cl, and $[\mathrm{BmPy}] \mathrm{Cl}$ could efficiently dissolve (more than $30 \mathrm{~g} / \mathrm{kg}$ solubility) and pretreat corn cob. After the five ILpretreated corn cob was enzymatically hydrolyzed for $24 \mathrm{~h}$, the reducing sugars were achieved in the concentrations of $3.55,3.63,3.47,3.63$, and $3.43 \mathrm{~g} / 1$, respectively. As shown in Fig. 1, using phosphate containing ILs or chloride-based ILs as pretreatment solvents, the conversions of corn cob to reducing sugars appeared to be at least two-fold than that of untreated one.

In the cellulase compatibility test, all ILs induced different degrees of effect on cellulase activity when comparing with the control experiment in acetate acidsodium acetate buffer. As shown in Fig. 2, when [Mmim] DMP, [DMEA][lactate], and DMEAA were added to the buffer in the ratio of $20 \%(v / v)$, respectively, no inhibition

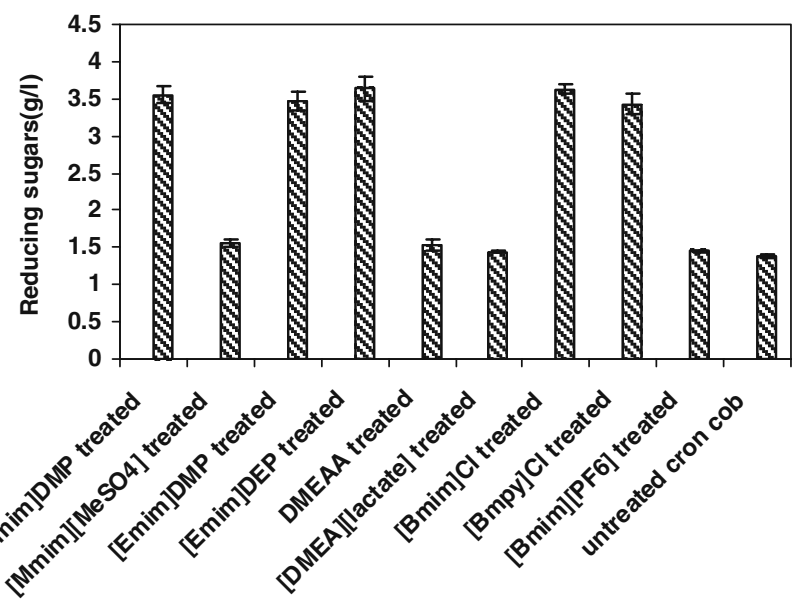

Fig. 1 Effect of ionic liquids pretreatment on enzymatic hydrolysis of pretreated corn cob. Pretreatment condition: the corn cob samples were incubated for $20 \mathrm{~min}$ at $130^{\circ} \mathrm{C}$ and regenerated using the antisolvent water. Enzymatic hydrolysis condition: regenerated corn cob and untreated corn cob $(10 \mathrm{~g} / \mathrm{l})$ were hydrolyzed using a cellulase activity of $30 \mathrm{FPU} / \mathrm{g}$ substrate at $50^{\circ} \mathrm{C}$ and $\mathrm{pH} 4.8$ for $24 \mathrm{~h}$

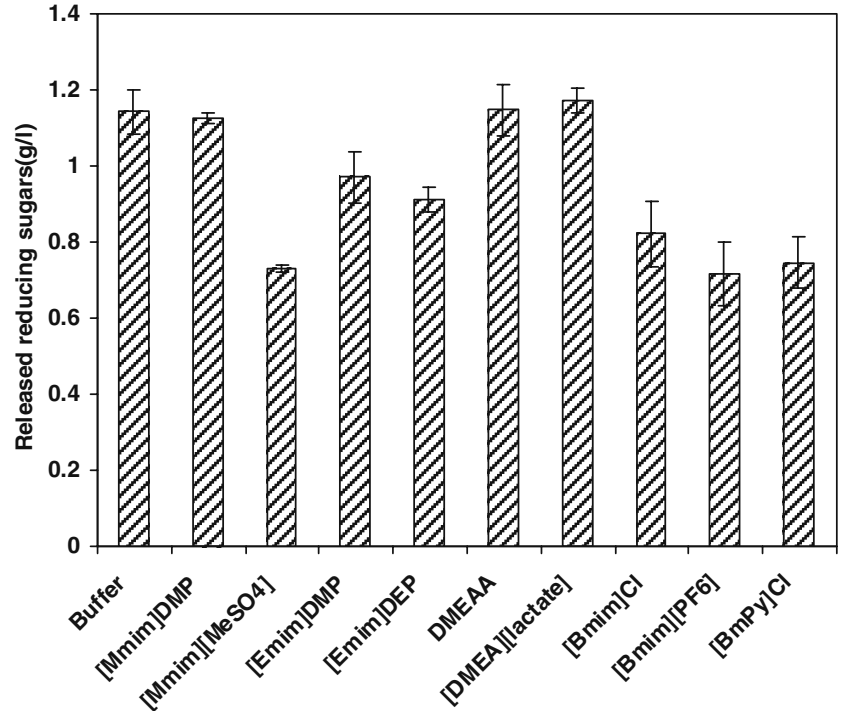

Fig. 2 Effect of ILs on the cellulase activity. Enzymatic hydrolysis of untreated corn cob powder $\left(50^{\circ} \mathrm{C}\right.$, reaction time $\left.3 \mathrm{~h}\right)$; cellulase activity in $20 \%(v / v)$ ILs were explored. After mixing the IL solution of $10 \mathrm{~g} / 1$ substrates (corn cob powder) with $50 \mathrm{mM}$ acetate acid-sodium acetate buffer ( $\mathrm{pH} 4.8$ ), 90FPU cellulase was directly added to the $3 \mathrm{ml} \mathrm{IL} /$ water mixtures at $50^{\circ} \mathrm{C}$ for $3 \mathrm{~h}$

on the cellulase activity was found. After enzymatic hydrolysis of untreated corn cob for $3 \mathrm{~h}$, the reducing sugar concentration in IL/buffer system was $1.13,1.17$, and $1.15 \mathrm{~g} / \mathrm{l}$, respectively vs. $1.14 \mathrm{~g} / 1$ in buffer (Fig. 2). However, when cellulase was exposed to $20 \%(v / v)$ [Emim] DMP or [Emim]DEP, little inhibition on the cellulase activity was found, and the yield of the reducing sugars was 0.97 and $0.91 \mathrm{~g} / \mathrm{l}$, respectively. On the other hand, the ILs [Mmim] $\left[\mathrm{MeSO}_{4}\right],[\mathrm{Bmim}] \mathrm{Cl},[\mathrm{Bmim}]\left[\mathrm{PF}_{6}\right]$, and $[\mathrm{BmPy}] \mathrm{Cl}$ mediated very low cellulase activity and supported the yield of 0.73 , $0.82,0.72$, and $0.75 \mathrm{~g} / \mathrm{l}$, reducing sugars in the untreated corn cob enzymatic hydrolysis.

Based on the above data, lignocellulose-dissolving IL [Mmim]DMP was chosen as a green solvent for corn cob pretreatment in view of its good biocompatibility with both lignocellulose solubility and cellulase activity. In addition, it is the first report that the IL [Mmim]DMP could dissolve cellulose or corn cob.

Evaluation of [Mmim]DMP pretreatment on enzymatic hydrolysis

As shown in the analytical methods, there was 44.8 wt.\% cellulose in regenerated corn cob. Furthermore, in the pretreatment/saccharification process (as shown in Fig. 3a), the released reducing sugars from corn cob pretreated with [Mmim]DMP at $130^{\circ} \mathrm{C}$ for 20 min reached $3.55 \mathrm{~g} / 1$ after being enzymatically hydrolyzed for $24 \mathrm{~h}$. Namely, after enzymatic hydrolysis, 79.3 wt.\% of theoretical yield of cellulose was hydrolyzed into reducing sugars, and $20.7 \%$ 

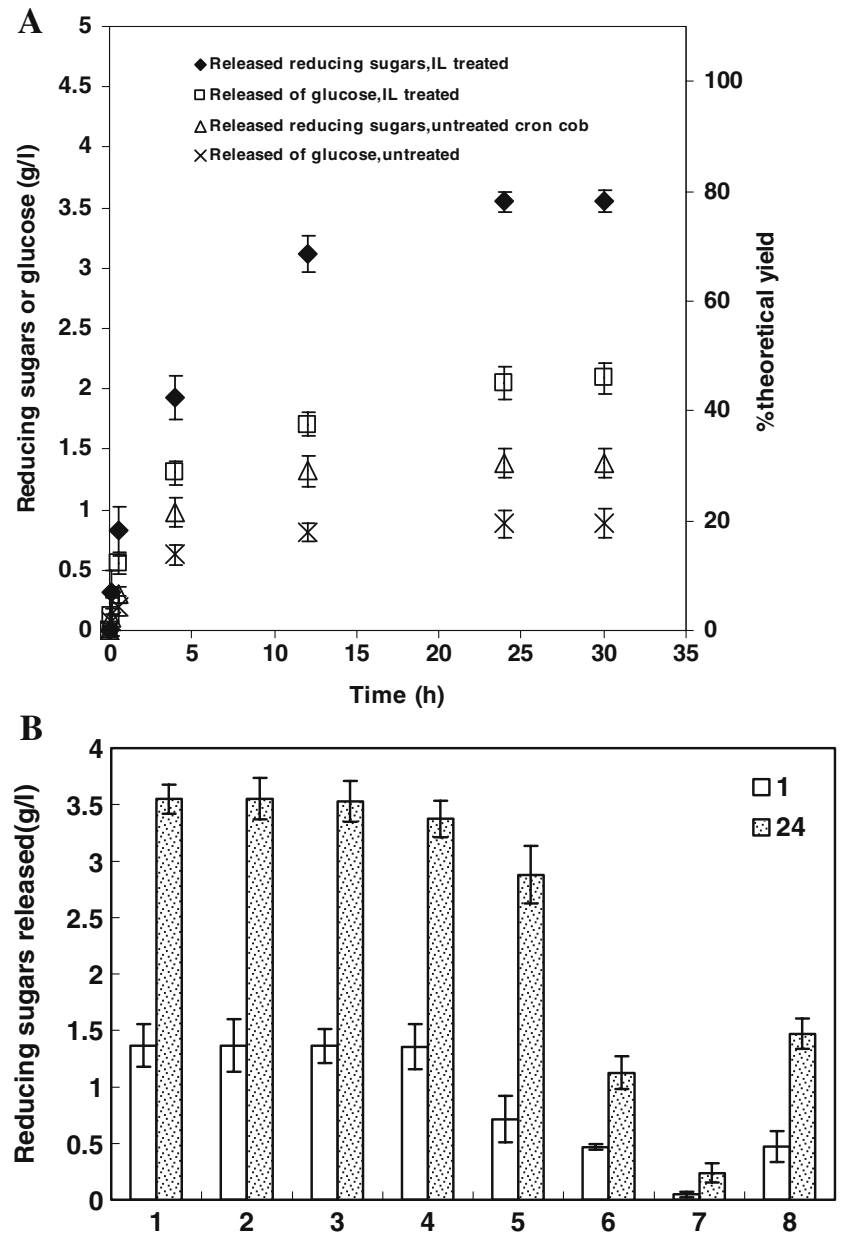

Fig. 3 a Profiles of enzymatic hydrolysis of corn cob regenerated from [Mmim]DMP. b Profiles of in situ enzymatic hydrolysis of corn cob in aqueous-[Mmim]DMP. Enzymatic hydrolysis condition with $10 \mathrm{~g} / 1$ regenerated corn cob, cellulase $30 \mathrm{FPU} / \mathrm{g}$ substrate, and $50^{\circ} \mathrm{C}$. (1) saccharification of pretreated corn cob in buffer, $(2 \sim 7)$ in situ saccharification of pretreated corn cob in $10,15,20,30,40,60 \%$ [Mmim]DMP, (8) saccharification of untreated corn cob in buffer

of cellulose was left in the solids. The data was similar to the enzymatic hydrolysis of untreated cellulose (20 wt.\%) and regenerated cellulose (80 wt.\%) data in Dadi and coworkers' report (Dadi et al. 2006).

In the enzymatic in situ hydrolysis process (as shown in Fig. $3 b)$, the cellulase activity in $10-20 \%(v / v)$ [Mmim] DMP was almost the same as enzymatic hydrolysis of regenerated corn cob in buffer in the first hour. After $24 \mathrm{~h}$ of saccharification, however, slightly reduced cellulase activity at $20 \%(v / v)$ [Mmim]DMP hydrolysis mixture was observed. In contrast, there was no negative effect on cellulase activity was observed in 15\% [Mmim]DMP enzymatic hydrolysis system. At the volume ratio of $15 \%$ [Mmim]DMP, reducing sugar formation $(3.56 \mathrm{~g} / \mathrm{l}$ reducing sugars) in the aqueous-IL mixture was approximately 2.3fold higher than enzymatic hydrolysis of untreated corn cob in the aqueous system in $24 \mathrm{~h}$, indicating that $15 \%$ [Mmim]
DMP would be the appropriate concentration to perform in situ saccharification process. As a result, the enzymefriendly IL can perform green process to obtain enhanced enzymatic saccharification.

To visualize the changes of regenerated corn cob structure, we recorded the infrared spectra and SEM images of untreated corn cob and pretreated corn cob. The FTIR spectra show characteristic cellulose peaks. The $1,437-\mathrm{cm}^{-1}$ band is for $\mathrm{CH}_{2}$ scissoring motion, and $899 \mathrm{~cm}^{-1}$ band (equivalent to $897 \mathrm{~cm}^{-1}$ absorbance) is for vibrational mode involving $\mathrm{C} 1$ and four atoms attached to it, which is characteristic of $\beta$ anomers or $\beta$-linked glucose polymers (Nelson and O'Connor 1964); the $1,378-\mathrm{cm}^{-1}$ band is for $\mathrm{C}-\mathrm{H}$ bending mode, and the 2,900- $\mathrm{cm}^{-1}$ band is for $\mathrm{C}-\mathrm{H}$ and $\mathrm{CH}_{2}$ stretching, which is unaffected by changes in crystallinity (Nelson and O'Connor 1964). From these spectra, two infrared ratios were calculated:

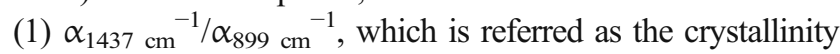
index (O'Connor et al. 1958) or lateral order index (LOI;

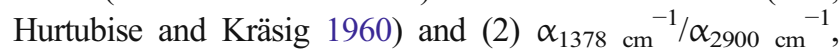
which is known as the total crystallinity index (TCI) (Nelson and O'Connor 1964). The higher index value represents that the material has a higher crystallinity and ordered structure. After pretreatment at $130^{\circ} \mathrm{C}$ for $20 \mathrm{~min}$, the LOI of corn cob decreased from 1.00 to 0.92 , and the TCI of corn cob significantly decreased from 1.05 to 0.88 . As a result, the regenerated corn cob, after dissolving in [Mmim]DMP, is less crystalline compared with the untreated one. SEM images of untreated and regenerated corn cob were displayed in Fig. 4. Compared with untreated corn cob (Fig. 4, 1a and 2a), more rupture and porous of fibers and more free of trenches were observed in corn cob regenerated from IL (Fig. 4, $1 \mathrm{~b}$ and 2b). Surface of corn cob granule showed that many terraces and steps form after IL pretreatment.

Evaluate the fermentability of hydrolysates by lipid fermentation

Subsequently, the fermentability of hydrolysates was evaluated by storage-lipid-accumulating bacterium $R$. opacus ACCC41043 to produce microbial lipid. As shown in Table 1 ("Hydrolyzates from pretreatment/saccharification process as carbon source"), $1.37 \mathrm{~g} / 1$ cell dry matter (CDM) and $41.02 \% \mathrm{CDM}$ lipid content were obtained after $30 \mathrm{~h}$ of cultivation using hydrolyzates (containing $3.55 \mathrm{~g} / \mathrm{l}$ sugars) from pretreatment/saccharification process. The accumulated lipid in R. opacus ACCC41043 had a fatty acid profile rich in $\mathrm{C} 16$ and $\mathrm{C} 18$ with four major constituent fatty acids (Table 2): n (oleic acid) $/ \mathrm{n}$ (stearic acid) $/ \mathrm{n}$ (palmitic acid) $/ \mathrm{n}$ (palmitoleic acid) $=22.46: 14.89: 24.74: 23.68$. In addition, high yield of $91.30 \%$, of fatty acid methyl esters (Table 1, "Hydrolyzates from pretreatment/saccharification process as carbon source") was obtained by esterification of the microbial lipid. 
Fig. 4 SEM image of untreated and ionic liquid-treated corn cob. $1 a$ untreated corn cob, $10.1 \mathrm{~mm} \times 5.00 \mathrm{k}$ SEM; $1 b$ [Mmim]DMP-treated corn cob, $10.1 \mathrm{~mm} \times 5.00 \mathrm{k} \mathrm{SEM} ; 2 a$ untreated corn cob, $10.1 \mathrm{~mm} \times$ $1.00 \mathrm{k}$ SEM, $2 b$ [Mmim]DMPtreated corn cob, $10.1 \mathrm{~mm} \times$ $1.00 \mathrm{k} \mathrm{SEM}$
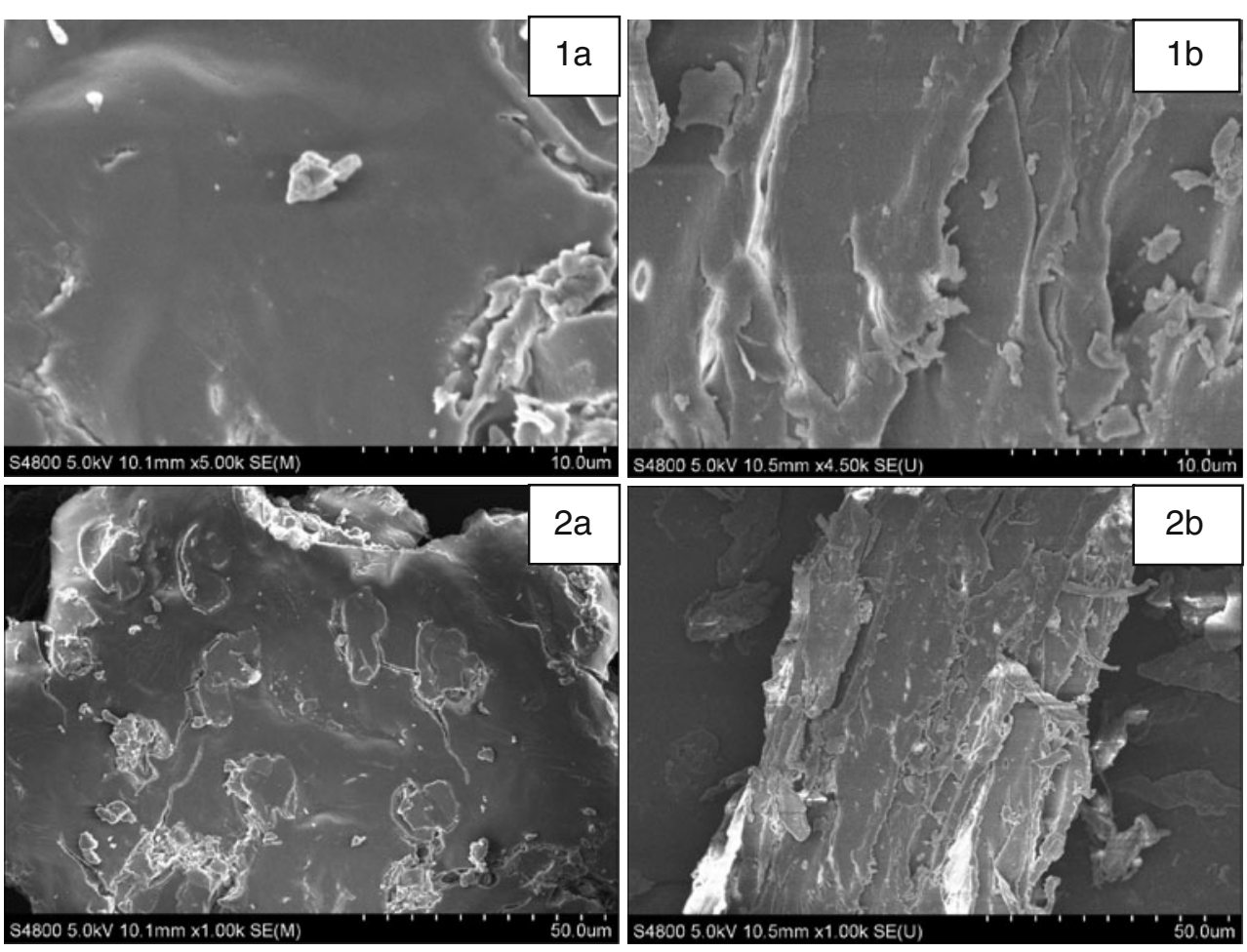

Hydrolyzates (containing $4.25 \mathrm{~g} / \mathrm{l}$ sugars) from enzymatic in situ hydrolysis process were also used as carbon source in MSM for microbial lipid fermentation. After $30 \mathrm{~h}$ of cultivation, lipid content were $42.86 \%$ CDM (Table 1, "Hydrolyzates from in situ saccharification process as carbon source") lipid with a fatty acid profile rich in C16 and $\mathrm{C} 18$ with four major constituent fatty acids: n(oleic acid $) / \mathrm{n}($ stearic acid $) / \mathrm{n}($ palmitic acid $) / \mathrm{n}($ palmitoleic acid $)=$ 23.46:15.68:22.82:18.93. Furthermore, high yield of $92.3 \%$ of fatty acid methyl esters (Table 1, "Hydrolyzates from in situ saccharification process as carbon source") was obtained by esterification of the microbial lipid. There was similar lipid accumulation and esterification rate of lipid between hydrolyzates and pure glucose. In contrast to the control test, there was no obvious negative effect observed in reducing sugar consumption and lipid production in the fermentation.

Furthermore, the effect of ILs on fermentation was investigated. As shown in Table 1 ("Hydrolyzates from in situ saccharification process as carbon source"), $1.63 \mathrm{~g} / \mathrm{l} \mathrm{CDM}$

Table 1 Lipid fermentation using hydrolyzates

\begin{tabular}{|c|c|c|c|}
\hline Carbon source & Lipid content $(\% \mathrm{CDM})$ & Biomass (g/l) & Yield of FAME (wt.\%) \\
\hline \multicolumn{4}{|c|}{ Hydrolyzates from pretreatment/saccharification process as carbon source } \\
\hline Control (3.55 g/l glucose) & 40.60 & 1.48 & 90.60 \\
\hline Hydrolyzate $^{\mathrm{a}}$ (3.55 $\mathrm{g} / 1$ reducing sugars) & 41.02 & 1.37 & 91.30 \\
\hline \multicolumn{4}{|c|}{ Hydrolyzates from in situ saccharification process as carbon source } \\
\hline Control (4.25 g/l glucose) & 42.60 & 1.62 & 92.6 \\
\hline Recovered sugars $^{\mathrm{b}}$ & 42.86 & 1.71 & 92.3 \\
\hline Recovered sugars $^{\mathrm{b}}$ with $10 \mathrm{~g} / 1$ [Mmim]DMP & 42.02 & 1.63 & 91.0 \\
\hline Recovered sugars $^{\mathrm{b}}$ with $15 \mathrm{~g} / 1$ [Mmim]DMP & 42.28 & 1.69 & 91.9 \\
\hline Recovered sugars $^{\mathrm{b}}$ with $10 \mathrm{~g} / 1[\mathrm{Bmim}] \mathrm{Cl}$ & - & 0.09 & - \\
\hline
\end{tabular}

\footnotetext{
${ }^{a}$ Hydrolyzate was from ionic liquid pretreatment/saccharification process

${ }^{\mathrm{b}}$ Reducing sugars (containing $4.25 \mathrm{~g} / 1$ reducing sugars) recovered from enzymatic in situ hydrolysis process

(En dash) not determined
}

R. opacus was cultivated for $30 \mathrm{~h}$ in low-nitrogen medium using hydrolyzates from ionic liquid pretreatment/saccharification process or in situ saccharification process as carbon source. Yield of FAME was measured by esterification using IL $N$-methyl-2-pyrrolidonium methyl sulfate $\left([\mathrm{NMP}]\left[\mathrm{CH}_{3} \mathrm{SO}_{3}\right]\right)$ as catalyst 
Table 2 Distribution of fatty acids in total fatty acids accumulated by R. opacus ACCC41043

\begin{tabular}{|c|c|c|c|c|}
\hline Carbon source & Palmitic acid & Palmitoleic acid & Stearic acid & Oleic acid \\
\hline Glucose (control) & 24.07 & 16.46 & 19.04 & 25.63 \\
\hline Hydrolyzates $^{\mathrm{a}}$ & 24.74 & 23.68 & 14.89 & 22.46 \\
\hline Recovered sugars ${ }^{\mathrm{b}}$ & 22.82 & 18.93 & 15.68 & 23.46 \\
\hline Recovered sugars $^{\mathrm{b}}$ with $10 \mathrm{~g} / 1 \mathrm{IL}$ & 22.67 & 19.55 & 16.72 & 23.15 \\
\hline Recovered sugars $^{\mathrm{b}}$ with $15 \mathrm{~g} / 1 \mathrm{IL}$ & 23.55 & 19.26 & 16.66 & 23.01 \\
\hline
\end{tabular}

${ }^{a}$ Hydrolyzate was from ionic liquid pretreatment/saccharification process

${ }^{\mathrm{b}}$ Reducing sugars recovered from enzymatic in situ hydrolysis process

IL: [Mmim]DMP

Cells were cultivated for $30 \mathrm{~h}$ in low-nitrogen medium using hydrolyzates as carbon source. The accumulated oil in $R$. opacus ACCC41043 with a fatty acid profile is rich in $\mathrm{C} 16$ and $\mathrm{C} 18$ with four major constituent fatty acids: oleic acid, palmitic acid, palmitoleic acid, and stearic acid

and $42.02 \%$ CDM lipid content were obtained after $30 \mathrm{~h}$ of cultivation using hydrolyzates (containing $4.25 \mathrm{~g} / 1$ reducing sugars) with the addition of $10 \mathrm{~g} / 1$ [Mmim]DMP. There was similar fatty acid profile between $R$. opacus culture from IL-free MSM and $R$. opacus culture from MSM with $10 \mathrm{~g} / 1$ [Mmim]DMP (Table 1, "Hydrolyzates from in situ saccharification process as carbon source"). Further increases of the IL to the concentration of $15 \mathrm{~g} / 1$ caused no obvious effect on CDM and lipid accumulation (Table 1, "Hydrolyzates from in situ saccharification process as carbon source"). In the contrast, the presence of $10 \mathrm{~g} / 1$ [Bmim] Cl could completely inhabit the growth of $R$. opacus ACCC41043.

In addition, the corn cob utilization for the overall microbial lipid production was also elucidated. In pretreatment/saccharification process and in situ saccharification process, $5.6 \%$ and $5.7 \%$ conversion rates were obtained, respectively.

\section{Discussion}

The pretreatment process is the key point in biomass conversion to biofuel, for lignocellulose must be pretreated prior to addition of hydrolytic enzymes for saccharification of cellulose, as yields are otherwise too low (Yang and Wyman 2008). Very recently, ILs showed promise as efficient novel solvents for pretreatment of cellulosic materials ( $\mathrm{Li}$ et al. 2009; Liu and Chen 2006). In addition, residue of ionic liquid from the pretreatment may follow the cellulose fibers to the enzymatic hydrolysis and fermentation process. Therefore, in our case, general profile of the biocompatibility of ionic liquid on cellulase, lipid production, fatty acid distribution, and cell growth of $R$. opacus were first studied.

Our finding of good cellulase compatibility in modest concentration $(20 \%, v / v)$ of phosphate-based ILs is in contrast to the strong deactivation or inhibition of cellulase when exposed to even low concentration of the chloridebased ILs $([\mathrm{Bmim}] \mathrm{Cl}$ and $[\mathrm{BmPy}] \mathrm{Cl})$ and vitriol-based IL ([Mmim $\left.]\left[\mathrm{MeSO}_{4}\right]\right)$ for cellulose dissolution and regeneration, probably because chloride- and vitriol-based ILs are corrosive and toxic (Turner et al. 2003; Zhao et al. 2007, 2008; Docherty and Kulpa 2005). Based on the enzymatic hydrolysis data in the presence of 20\% [Mmim]DMP, [DMEA][lactate], or DMEAA, the conversion of corn cob to glucose was similar to that of buffer. Among these ILs, DMEAA and [DMEA][lactate] showed good biocompatibility, but they do not dissolve cellulose or corn cob.

In the cellulosic material pretreatment test, imidazolium and pyridinium cation-based ILs possessed a wide range of MCC and corn cob solubility depending on the associated anion. [Mmim]DMP, [Emim]DMP, [Emim]DEP, [Bmim] $\mathrm{Cl}$, and $[\mathrm{BmPy}] \mathrm{Cl}$ were effective in dissolving cellulose instead of DMEAA, [DMEA][lactate], and [Mmim] $\left[\mathrm{MeSO}_{4}\right]$. In addition, Zhao and coworkers reported that [Bmim][OAc] can dissolve cellulose (Zhao et al. 2008). The corn cob regenerated from [Emim]DMP and [Emim]DEP showed similar saccharification rate to corn cob regenerated from [Mmim]DMP, but the cellulase compatibility of the former two ILs was lower than [Mmim]DMP. In conclusion, both the cation and anion in ILs mediated the important advantage of lignocellulose-dissolving ILs as good solvent for lignocellulose materials. In addition, our data suggested that only cellulose-dissolving ILs could conduct efficient corn cob pretreatment and result in pretreated corn cob-enhanced enzymatic hydrolysis.

Compared with other phosphate-based ILs and chloridebased ILs investigated, [Mmim]DMP was chosen as a green solvent to pretreat corn cob in view of its biocompatibility with both lignocellulose pretreatment and cellulase activity. Both the [Mmim]DMP involved pretreatment/ saccharification process and in situ saccharification process could efficiently convert corn cob to sugars which is important from the practical viewpoint. As a result, the 
small amount residue of the IL from the IL pretreatment process will pose no negative effect on enzymatic hydrolysis. Moreover, the IL can be easily synthesized using lowcost raw materials for large-scale manufacture (Nie et al. 2006). For these reasons, our work focused on the use of this IL [Mmim]DMP as pretreatment solvent in the study for the efficient enzymatic hydrolysis of corn cob. Fifteen percent $(v / v)$ [Mmim]DMP would be the appropriate solution to perform enzymatic in situ saccharification of corn cob, for there would be inhabit cellulase activity in $20 \%$ or higher concentration of [Mmim]DMP after $24 \mathrm{~h}$ of enzymatic hydrolysis (Fig. 3b).

Based on the FTIR data, the decreases of LOI and TCI indexes had been similarly reported for wheat straw regenerated from IL [Bmim]Cl dissolution (Liu and Chen 2006). According to the SEM data, it is evident that the fibers were partially disrupted, instead of clearly isolated, individual fibers, which are characteristic for untreated corn cob. Probably, during the process of dissolution and following recovery procedure, the lignocellulosic material has lost its crystalline structure and, instead, restructured itself into a mostly amorphous form (Mikkola et al. 2007). Consequently, SEM image showed that the fragmental and porous regenerated corn cob with amorphous structure could provide more surfaces for enzymes to attack on. As a result, combined with the enzymatic hydrolysis profile of regenerated corn cob (Fig. 2), FTIR analysis and SEM image of corn cob regenerated, it could be concluded that the intact structure was disrupted by IL [Mmim]DMP pretreatment and resulted in a porous and amorphous regenerated corn cob that greatly enhanced enzymatic hydrolysis.

The hydrolyzates could be subsequently transformed into biofuel by microbial fermentation. In our case, the lipid fermentations were performed by $R$. opacus ACCC41043 using hydrolyzates (containing reducing sugars) as carbon source. Our data suggest that the hydrolyzates (from pretreatment/saccharification process or enzymatic in situ hydrolysis process) posed no negative effect on $R$. opacus ACCC41043 growth and lipid production. It was reported that $R$. opacus can accumulate lipid up to $20-76 \%$ of the CDM in nitrogen-limited medium (Alvarez et al. 1997; Steinbüchel 2001), while our data suggested that the $R$. opacus strain ACCC 41043 could accumulate $41-43 \%$ lipid of CDM using simple mineral salt medium with hydrolyzates as carbon source.

It was reported by Alvarez and coworkers that the $\mathrm{C} 16$ and $\mathrm{C} 18$ distribution in $R$. opacus MR22 are 34.8 and $39.3 \mathrm{~mol} \%$, respectively (Alvarez et al. 1997). The composition of $\mathrm{C} 18$ in $R$. opacus ACCC41043 was similar to that of $\mathrm{C} 18$ in $R$. opacus MR22. The concentration of C16 in $R$. opacus ACCC41043 was $8.9 \mathrm{~mol} \%$ higher than that of $R$. opacus MR22. In addition, there was similar distribution of fatty acids in total fatty acids accumulated by
$R$. opacus ACCC41043 using hydrolyzates or glucose as carbon source (Table 1, "Hydrolyzates from pretreatment/ saccharification process as carbon source"). The main composition of $R$. opacus ACCC41043 lipid, which was abundant in $\mathrm{C} 16$ and $\mathrm{C} 18$, contained long-chain fatty acids that are comparable to conventional vegetable oils ( $\mathrm{Li}$ et al. 2007). Furthermore, there was no obvious negative effect in the cell growth and lipid accumulation of $R$. opacus ACCC41043 when exposed to the MSM with 10-15 g/ 1 IL [Mmim]DMP. On all accounts, these evidences indicated that corn cob pretreated by IL [Mmim]DMP did not pose obvious negative effects on the growth and lipid production of $R$. opacus ACCC41043. In addition, the microbial lipid was easily esterificated into biodiesel (fatty acid alkyl esters, FAME) with high yield $91-93 \%$ of FAME using IL [NMP] $\left[\mathrm{CH}_{3} \mathrm{SO}_{3}\right]$ as catalyst. As a result, the IL mediated green process of bioconversion of low-cost lignocellulosic materials into biodiesel is promising for biofuel production and the environmental pollution control.

In summary, our work elucidated that IL [Mmim]DMP mediated the obvious advantage as biocompatible solvent for lingnocellulose pretreatment. The pretreatment/saccharification process and in situ saccharification process involving [Mmim]DMP were efficiently performed in bioconversion of corn cob to sugars, and more than $70 \%$ saccharification rates were obtained in $24 \mathrm{~h}$. Our data also suggested that the hydrolysis products did not pose negative effects on lipid production and accumulation of $R$. opacus ACCC41043. The microbe could efficiently accumulate lipid up to $41-43 \%$ CDM using hydrolyzates. Such information will be vital for tackling the hydrolysis of more complex lignocellulosic materials and biotransform hydrolyzates obtained from enzymatic hydrolysis of ILtreated cellulose to biofuel.

Acknowledgements This work was financially supported by CAS 100 Talents Program (KGCX2-YW-801). We are grateful to Dr. Ling Hua and Bo Li for their kindly donation of cellulase (Genencor).

\section{References}

Alvarez HM, Kalscheuer R, Steinbüchel A (1997) Accumulation of storage lipids in species of Rhodococcus and Nocardia and effect of inhibitors and polyethylene glycol. FeW Lipid 99:239-246

Chandra RP, Bura R, Mabee WE, Berlin A, Pan X, Saddler JN (2007) Substrate pretreatment: the key to effective enzymatic hydrolysis of lignocellulosics. Adv Biochem Eng Biotechnol 108:67-93

Dadi AP, Varanasi S, Schall CA (2006) Enhancement of cellulose saccharification kinetics using an ionic liquid pretreatment step. Biotechnol Bioeng 95:904-910

Diego AF, Richard CR, Richard PS, Patrick M, Guillermo M, Robin DR (2007) Can ionic liquids dissolve wood? Processing and analysis of lignocellulosic materials with 1-n-butyl-3-methylimidazolium chloride. Green Chem 9:63-69 
Docherty KM, Kulpa CF (2005) Toxicity and antimicrobial activity of imidazolium and pyridinium ionic liquids. Green Chem 7:85-189

Galbe M, Zacchi G (2007) Pretreatment of lignocellulosic materials for efficient bioethanol production. Adv Biochem Eng Biotechnol 108:41-65

Goering HK, Van Soest P (1970) Forage fibre analysis. Apparatus, reagents, procedure and some applications. Agric Handbook 379, Washington DC, pp 1-20, ARS. USDA

Hendriks A, Zeeman G (2009) Pretreatments to enhance the digestibility of lignocellulosic biomass. Bioresour Technol 100:10-18

Hélène OB, Lionel M (2002) Ionic liquids: perspectives for organic and catalytic reactions. J Mol Catal A Chem 182-183:419-437

Heinze T, Liebert T (2001) Unconventional methods in cellulose functionalization. Prog Polym Sci 26:1689-1762

Huddleston JG, Visser AE, Reichert WM, Willauer HD, Broker GA, Rogers RD (2001) Characterization and comparison of hydrophilic and hydrophobic room temperature ionic liquids incorporating the imidazolium cation. Green Chem 3:156-164

Hurtubise FG, Kräsig H (1960) Classification of fine structural characteristics in cellulose by infrared spectroscopy. Use of potassium bromide pellet technique. Anal Chem 32:177-181

Kamiya N, Matsushita Y, Hanaki M, Nakashima K, Narita M, Goto M, Takahashi H (2008) Enzymatic in situ saccharification of cellulose in aqueous-ionic liquid media. Biotechnol Lett 30:1037-1040

Li Q, He YC, Xian M, Jun G, Xu X, Jian MY, Li LZ (2009) Improving enzymatic hydrolysis of wheat straw using ionic liquid 1-ethyl-3-methyl imidazolium diethyl phosphate pretreatment. Bioresour Technol 100:3570-3575

Li YH, Zhao ZB, Bai FW (2007) High-density cultivation of oleaginous yeast Rhodosporidium toruloides $\mathrm{Y} 4$ in fed-batch culture. Enzyme Microb Technol 41:312-331

Liu LY, Chen HZ (2006) Enzymatic hydrolysis of cellulose materials treated with ionic liquid [Bmim]Cl. Chin Sci Bull 51:2432-2436

Long ZD, Xu JH, Pan J (2007) Immobilization of Serratia marcescens lipase and catalytic resolution of trans-3-(4'-methoxyphenyl) glycidic acid methyl ester. Chin J Catal 28:175-179

Miller GL (1959) Use of dinitrosalicylic acid reagent for determination of reducing sugar. Anal Chem 31:426-428

Mikkola JP, Kirilin A, Tuuf JC, Pranovich A, Holmbom B, Kustov LM, Murzin DY, Salmi T (2007) Ultrasound enhancement of cellulose processing in ionic liquids: from dissolution towards functionalization. Green Chem 9:1229-1237

Mosier N, Wyman C, Dale B, Elander R, Lee YY, Holtzapple M, Ladisch M (2005) Features of promising technologies for pretreatment of lignocellulosic biomass. Bioresour Technol 96:73-686

Nelson ML, O'Connor RT (1964) Relation of certain infrared bands to cellulose crystallinity and crystal lattice type. Part II. A new infrared ratio for estimation of crystallinity in celluloses I and II. J Appl Polym Sci 8:1325-1341

Nie Y, Li C, Sun A, Meng H, Wang Z (2006) Extractive desulfurization of gasoline using imidazolium-based phosphoric ionic liquids. Energ Fuels 20:2083-2087

O’Connor RT, DuPré EF, Mitcham D (1958) Applications of infrared absorption spectroscopy to investigations of cotton and modified cottons. Part I: physical and crystalline modifications and oxidation. Tex Res J 28:382-392

Peterson A, Thomsen MH, Nielsen HH, Thomsen AB (2007) Potential bioethanol and biogas production using lignocellulosic biomass from winter rye, oilseed rape and faba bean. Biomass Bioenergy 31:812-819

Rayne S, Mazza G (2007) Trichoderma reesei derived cellulase activity in three $N, N$-dimethylethanolammonium akylcarboxylate ionic liquids. Nat Precedings p 1-17 (http:/hdl.nature.com/ 10101/npre.2007.632.1)

Rogers RD, Seddon KR (2003) Ionic liquids - solvents of the future? Science 302:792-793

Roosen C, Müller P, Greiner L (2008) Ionic liquids in biotechnology: applications and perspectives for biotransformations. Appl Microbiol Biotechnol 81:607-614

Rubin EM (2008) Genomics of cellulosic biofuels. Nature 45:841-845

Schlegel HG, Kaltwasser H, Gottschalk G (1961) Ein Submersverfahren zur Kultur wasserstoffoxydierender Bakterien: wachstumsphysiologische Untersuchungen. Arch Mikrobiol 38:209-222

Schmid A, Kollmer A, Mathys RG, Withot B (1998) Development toward large-scale bacterial bioprocesses in the presence of bulk amounts of organic solvents. Extremophiles 2:249-256

Steinbüchel IVA (2001) High cell density cultivation of Rhodococcus opacus for lipid production at a pilot-plant scale. Appl Microbiol Biotechnol 55:547-555

Sun Y, Cheng JY (2002) Hydrolysis of lignocellulosic materials for ethanol production: a review. Bioresour Technol 83:1-11

Turner MB, Spear SK, Huddleston JG, Holbrey JD, Rogers RD (2003) Ionic liquid salt-induced inactivation and unfolding of cellulase from Trichoderma reesei. Green Chem 5:443-447

Wackett LP (2008) Biomass to fuels via microbial transformations. Curr Opin Chem Biol 12:187-193

Wältermann M, Luftmann H, Baumeister D, Kalscheuer R, Steinbüchel A (2000) Rhodococcus opacus strain PD630 as a new source of high-value single-cell oil? Isolation and characterization of triacylglycerols and other storage lipids. Microbiol 146:1143-1149

Xian M, Li LZ, He YC, Tan WQ, Li Q, Yang F (2009) In situ enzymatic hydrolysis of cellulose and the recovery method for ionic liquid and glucose. Chinese Patent, Application No. 200910093300.8

Yang B, Wyman CE (2008) Pretreatment: the key to unlocking lowcost cellulosic ethanol. Biofuels Bioproducts Bioref 2:26-40

Zhang YH, Lynd LR (2004) Toward an aggregated understanding of enzymatic hydrolysis of cellulose: noncomplexed cellulase systems. Biotechnol Bioeng 88:797-824

Zhang L, Ji J, Deng D, Shu Z, Chen X (2008) Preparation of one kind of acetate based ionic liquid. Chinese Patent, CN, 101108827A

Zhang L, Xian M, He YC, Li LZ, Yang JM, Yu ST, Xu X (2009) A Brønsted acidic ionic liquid as an efficient and environmentally benign catalyst for biodiesel synthesis from free fatty acids and alcohols. Bioresour Technol 100:4368-4373

Zhao H, Lee J, Song ZY, Olarongbe O (2006) Enhancing protease enantioselectivity by ionic liquids based on chiral- or $\omega$-amino acids. Tetrahedron Asymmetry 17:1549-1553

Zhao H, Gary AB, Zhi YS, Olarongbe O, Tanisha C, Darkey SP (2008) Designing enzyme-compatible ionic liquids that can dissolve carbohydrates. Green Chem 10:696-705

Zhao H, Cecil LJ, Gary AB, Shu QX, Olarongbe O, Vernecia NP (2009) Regenerating cellulose from ionic liquids for an accelerated enzymatic hydrolysis. J Biotech 139:47-54

Zhao DB, Liao YC, Zhang ZD (2007) Toxicity of Ionic Liquids. CLEAN Soil Air Water 35:42-48 